\title{
Teoría del Conectivismo en el Proceso de Aprendizaje en Red de la Respiración Celular
}

\section{Theory of Connectivism in the Network Learning Process of Cell Respiration}

Juan Diego Wilches-Vega ${ }^{1}$ \section{EDICIÓN: Reciliencia}

Recibido: 16/julio/2021

OAceptado: 17/octubre/2021

Publicado: 29/noviembre/2021

Páginas: $143-150$

(1) País

${ }^{1}$ Colombia

\section{IIIII Institución}

${ }^{1}$ Universidad de Santander

\section{Correo Eletrónico}

1juan.wilches@unipamplona.edu.co

\section{(D) ORCID}

'https://orcid.org/0000-0003-1067-4079

\section{Citar así: C APA / IEEE}

Wilches-Vega, J. (2021). Teoría del Conectivismo en el Proceso de Aprendizaje en Red de la Respiración Celular. Revista Tecnológica-Educativa Docentes 2.0, 1(1), 143-150

https://doi.org/10.37843/rted.v1i1.264

J. Wilches-Vega, "Teoría del Conectivismo en el Proceso de Aprendizaje en Red de la Respiración Celular", RTED, vol. 1, n. ${ }^{\circ}$, pp. 143-150, nov. 2021.

\section{Resumen}

El ciclo básico de los programas de medicina se compone de asignaturas caracterizadas por ser enseñadas de manera tradicionalista, presentar contenidos complejos y en constante actualización, generando dificultades y rechazo en los aprendices. Por este motivo, el presente trabajo tuvo como objetivo analizar la incidencia del conectivismo de George Siemens en el proceso de aprendizaje en red en los cursos de Bioquímica y Biología Molecular, pues esta teoría propone herramientas para mejorar el aprendizaje de los estudiantes, a través de su inmersión, navegación y construcción de redes de aprendizaje atractivas, autogestionadas y mediadas por tecnología. Se realizó una investigación de corte cualitativo, basado en la metodología de investigación acción, el aprendizaje basado en problemas y características propias del conectivismo, con estudiantes de pregrado de primer semestre de Medicina. La población considerada en esta investigación fueron estudiantes de las carreras del área de la salud que, dentro de sus cursos formativos, debían cursar asignaturas relacionadas con bioquímica y biología molecular. Se aplicaron cuestionarios de entrada y de salida, de y se aplicaron entrevistas a los participantes, como parte del diseño de una unidad didáctica, encaminada hacia la construcción de Blogs sobre la respiración celular, estudiada desde diversos procesos fisiológicos humanos. El conectivismo facilitó y motivó a los estudiantes a explorar las herramientas que tiene a su disposición y desconoce, los invitó a construir su propia red de aprendizaje y a gestionar su proceso formativo.

Palabras clave: Conectivismo, aprendizaje en red, bioquímica, biología molecular.

\section{Abstract}

The basic cycle of medicine programs comprises subjects characterized by being taught in a traditionalist way, presenting complex and constantly updated content, generating difficulties and rejection in the apprentices. For this reason, the present work aimed to analyze the incidence of George Siemens' connectivism in the network learning process in Biochemistry and Molecular Biology courses. This theory proposes tools to improve student learning through immersion, navigation, and construction of attractive, selfmanaged, and technology-mediated learning networks. Connectivism facilitated and motivated students to explore the tools they have at their disposal and are unknown to, invited them to build their learning network, and manage their training process. Qualitative research was carried out, based on the action research methodology, problem-based learning, and characteristics of connectivism, with undergraduate students of the first semester of Medicine. The population considered in this research were students from health careers who, within their training courses, had to take subjects related to biochemistry and molecular biology. Entry and exit questionnaires were applied, and interviews were used with the participants to design a didactic unit aimed at the construction of Blogs on cellular respiration, studied from various human physiological processes.

Keywords: Connectivism, network learning, biochemistry, molecular biology. 


\section{Introducción}

El ciclo básico de los programas de medicina se compone de asignaturas caracterizadas por ser enseñadas de manera tradicionalista, presentar contenidos complejos y en constante actualización, generando dificultades y rechazo en los aprendices. Por una parte, debido a que sus contenidos no se presentan de una manera atractiva para ellos y por otra, debido a que no logran identificar la relevancia para su futuro profesional, pues se encuentran desconectadas académicamente (Wood, 2001). Con el fin de aprobar esas asignaturas, los estudiantes se dedican a memorizar en lugar de comprender y entender lo estudiado. En consecuencia, se presentan bajos rendimientos académicos, poco interés, muy baja retención de información y poca apropiación del conocimiento. Particularmente, las asignaturas de bioquímica y biología molecular han sido objeto de revisión frente a estas situaciones.

En los 50's y 60's fue fundamental memorizar conceptos y procesos bioquímicos y moleculares, pues grandes descubrimientos y avances en estas áreas se encontraban en auge (Wood, 2001). Para los años 70 y 80, clases magistrales, prácticas y actividades en pequeños grupos de trabajo sirvieron para mejorar la enseñanza de bioquímica y biología molecular, sin embargo, Campbell (1975), compiló las críticas sobre el papel de la bioquímica como componente formativo de los estudiantes de medicina. Se destacó la falta de pertinencia y relación con su formación profesional, el excesivo tiempo de dedicación requerido para los componentes teóricos-prácticos, la complejidad y abstracción de sus contenidos. Posteriormente, Sequeira (1988) presentó los metabologramas como apoyo de estudio de esta asignatura y encontró que sus estudiantes los consideraron relevantes, pertinentes y útiles.

El elevado costo de los equipos especializados para estudiar los procesos metabólicos y moleculares, así como el alto nivel de formación que se requiere en quienes los utilizan, dificulta su aprendizaje a través de la observación directa o de actividades prácticas (Lucumí-Moreno, 2015). El conectivismo, planteado por George Siemens (2005), puede aportar soluciones, pues plantea que, la disposición de la información, la velocidad de actualización de los conocimientos y las características de los estudiantes han cambiado. La creatividad, la conexión con colaboradores, así como, el conocer de donde obtener información adecuada y confiable, son competencias fundamentales para los nuevos alumnos. El apoyo de las Tecnologías de la Información y la Comunicación (TIC) y las Tecnologías del Aprendizaje y el Conocimiento (TAC) es fundamental y beneficioso. Las redes, pueden ser definidas como conexiones entre seres. Las redes de computadores, las redes sociales, entre otras funcionan con el mismo principio, donde las personas, los grupos, los nodos, sistemas y entidades pueden estar conectadas para crear un todo (Siemens, 2005).

En ese contexto, donde se consolida una economía basada en el conocimiento, se vuelve clave tener la capacidad de acceder y usar la información almacenada en las redes (Rodrigo-Mendizábal et al., 2020), es necesaria una transformación e innovación en la enseñanza y el aprendizaje de los procesos bioquímicos y moleculares para mejorar su comprensión, apropiación y especialmente para despertar mayor interés en los aprendientes. Por lo anterior, el objetivo general de esta investigación fue analizar la incidencia de la teoría del conectivismo de George Siemens en el proceso de aprendizaje en red en los cursos de Bioquímica y Biología Molecular.

\section{Metodología}

Esta investigación se fundamentó bajo el paradigma cualitativo ya que se buscó explorar, describir e interpretar un fenómeno a partir de las experiencias individuales de los participantes; particularmente para analizar la incidencia de la teoría del conectivismo de George Siemens en el proceso de aprendizaje en red en los cursos de Bioquímica y Biología Molecular. En este proceso, los participantes se comportaron como lo hacen de manera 
cotidiana en sus clases, tal como lo sugiere Hernández-Sampieri et al. (2006), conociendo, explorando e interactuando con aspectos conectivistas inmersos en su proceso de aprendizaje.

La investigación cualitativa cuenta con un gran número de estrategias metodológicas que llevan a alcanzar los objetivos de cualquier investigación. Dadas las características del presente trabajo, el diseño metodológico planteado incluye aportes desde la investigación acción, como herramienta clave para estudiar la realidad educativa, acercarse e interpretar los fenómenos en campo; el aprendizaje basado en problemas, pues es uno de los pilares de la formación en el programa de Medicina de la Universidad de Santander; y tanto métodos, como herramientas, propias del conectivismo a saber, el uso de aulas virtuales, creación de blogs, navegación de redes sociales, entre otras herramientas multimediales para interactuar en línea.

En primer lugar, se propone la situación problema o el núcleo problemático sobre el cuál trabajarán los estudiantes (Muñoz-Cano et al., 2011), como una estrategia para desarrollar la creatividad y el trabajo en equipo (Saldaña, 2021). En este caso, se trabajó en torno a la respiración celular, un proceso que hace parte de las rutas metabólicas humanas y que está íntimamente ligado con la producción de energía en las células humanas para el correcto funcionamiento de todos los sistemas del cuerpo. Los alcances pueden ser mayores al relacionar la respiración celular con otros procesos, con momentos específicos del cuerpo, enfermedades, e incluso componentes ambientales como el aire y sus contaminantes.

En ese orden de ideas, se organizaron grupos de trabajo; se sugirieron temas generales en torno a la respiración celular, con el fin de despertar la curiosidad de los estudiantes, motivarlos a iniciar con su investigación y a perfilar el tema de acuerdo con sus intereses y experiencias particulares; se les invitó a realizar una breve búsqueda de cada uno de esos temas con el fin de seleccionar su preferido basados en una decisión informada. Los tópicos seleccionados por los grupos de trabajo fueron: La respiración celular y su influencia en los deportistas de alto rendimiento; La respiración celular y sus características en células cancerígenas (El efecto Warburg) (Koppenol et al., 2011); y El efecto de los contaminantes del aire en la respiración humana.

En segundo lugar, debe planearse el tipo de producto que se obtendrá al finalizar el proceso (Helle et al., 2006) por lo que se plantearon dos productos como mínimo. El primero, consistió en la elaboración de una monografía en torno al tópico seleccionado por cada grupo de trabajo, presentada en estilo de artículo de revisión. El segundo, fue la elaboración de un material en sintonía con los planteamientos de Siemens $(2004,2007)$ y Downes $(2005,2006,2010)$, un producto construido con mayor uso de herramientas conectivistas. Se sugirió elaborar de un blog para la divulgación de la información recopilada en torno a su temática específica, al que puedan acceder otros individuos desde cualquier lugar del mundo e interactuar con los autores (Altamirano, 2010).

La población considerada en esta investigación abarca a aquellos estudiantes de las carreras del área de la salud que, dentro de sus cursos formativos, deban cursar asignaturas relacionadas con bioquímica $\mathrm{y}$ biología molecular, pues son asignaturas que presentan dificultades para los estudiantes, especialmente por su alta complejidad y por ser altamente memorísticas. Adicionalmente, se entiende que los estudiantes universitarios deben tener ciertas competencias básicas relacionadas con el aprendizaje conectivista, como autonomía, interactividad, apertura y diversidad (Bernal, 2019).

Estas características las cumplen los estudiantes, teniendo en cuenta sus competencias cognitivas, actitudinales $\mathrm{y}$ tecnológicas, las cuales son clave para el uso esperado de las TIC (Valerio-Ureña \& Valenzuela-González, 2011). En segundo lugar, la muestra o informantes clave estuvieron conformados por estudiantes de la asignatura de Biociencias I, del Programa de Medicina de la Universidad de Santander Campus Cúcuta. Específicamente, se trabajó 
con 16 estudiantes, quienes desarrollaron su proyecto sobre la respiración celular.

Los instrumentos implementados en esta investigación se diseñaron siguiendo los principios conectivistas de aprendizaje en red, navegación y mantenimiento de la red, autogestión y autoaprendizaje. Corresponden a herramientas elaboradas para extraer información que permita responder a los objetivos planteados y así analizar, el impacto de la teoría del conectivismo en el proceso de aprendizaje en red de los estudiantes de primer semestre de medicina, particularmente de las áreas de bioquímica y biología molecular.

Para este fin, se aplicó una prueba de entrada y una prueba de salida a los estudiantes que hicieron parte del estudio, respecto a sus conocimientos generales sobre el proceso de respiración celular y su relación con el proyecto seleccionado. Además, se realizaron dos entrevistas a los estudiantes participantes, enfocada en identificar sus habilidades, sus competencias y su disposición hacia el uso de las herramientas tecnológicas de la web 2.0 como parte de su ecosistema de estudio $\mathrm{y}_{\overline{5}}$ además, para identificar cambios tras su exposición a técnicas conectivistas. Es importante mencionar que, dadas las condiciones de conectividad y aislamiento, los cuestionarios fueron respondidos por 9 estudiantes y las entrevistas se aplicaron a 5 estudiantes.

Adicional a los instrumentos aplicados, se realizó un diseño didáctico como carta de navegación para el desarrollo del proyecto de investigación en el cual los estudiantes pudiesen explorar la respiración celular aplicada al desarrollo de una enfermedad o relacionada con algún proceso físiológico normal del cuerpo. Este diseño didáctico contó con 11 sesiones organizadas en 5 etapas. En cada uno de los encuentros se trabajó en torno del desarrollo del proyecto mencionado y se expusieron las herramientas conectivistas para el aprendizaje a los estudiantes. Finalmente, el análisis de los resultados se realizó con base en la definición de conceptos y categorías establecidos por Strauss \& Corbin (2002).

\section{Resultados}

La implementación del conectivismo impactó positivamente el proceso de aprendizaje de bioquímica y biología molecular, específicamente de respiración celular en el grupo de estudiantes del programa de Medicina de la Universidad de Santander, Campus Cúcuta a través de la elaboración de artículos científicos y blogs de divulgación científica. Se evidenciaron mejoras en el rendimiento académico $(+5 \%)$ y mayor apropiación y uso de herramientas de la web 2.0, así como mayor reconocimiento a la construcción de redes de aprendizaje personales, tal como se evidencia en el conectivismo (ver Figura 1).

\section{Figura 1}

Teoría del Conectivismo

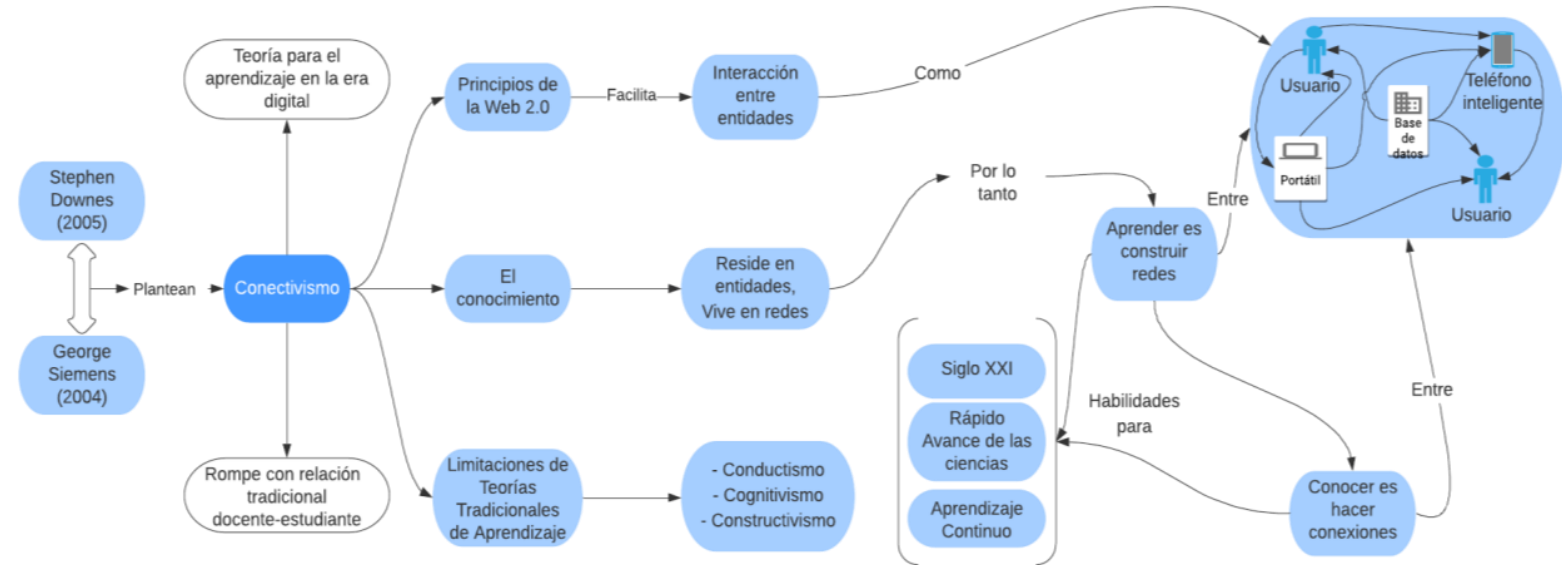

Nota. Experiencia de aprendizaje a través del conectivismo, elaboración propia (2021). 
Durante la implementación de la unidad didáctica diseñada, se aplicaron 9 pruebas de entrada y 9 de salida, las cuales evidenciaron progreso significativo en cuanto al proceso de aprendizaje de respiración celular, como componente fundamental de los cursos de bioquímica y biología molecular del programa de medicina de la Universidad de Santander, Campus Cúcuta. Se pudo observar en la Figura 2, que 3 estudiantes obtuvieron el $100 \%$ de respuestas correctas en el cuestionario de salida, mientras que, en el cuestionario de entrada, ninguno alcanzó dicho puntaje. También es importante resaltar que, a nivel general, se pasó de un $76 \%$ de respuestas correctas a un $81 \%$, lo que significa, un 5\% de mejora en el aprendizaje de la respiración celular. Pese a esto, 2 estudiantes no obtuvieron resultados satisfactorios en la prueba de salida e incluso, disminuyeron respecto a su prueba de entrada. Sin embargo, esta situación la atribuyen los estudiantes a dificultades de conexión que experimentaron al momento de presentar el cuestionario.

\section{Figura 2}

\section{Puntajes Obtenidos en los Cuestionarios de Entrada y de Salida por cada Estudiante}

\section{Calificación Cuestionarios por Estudiante}

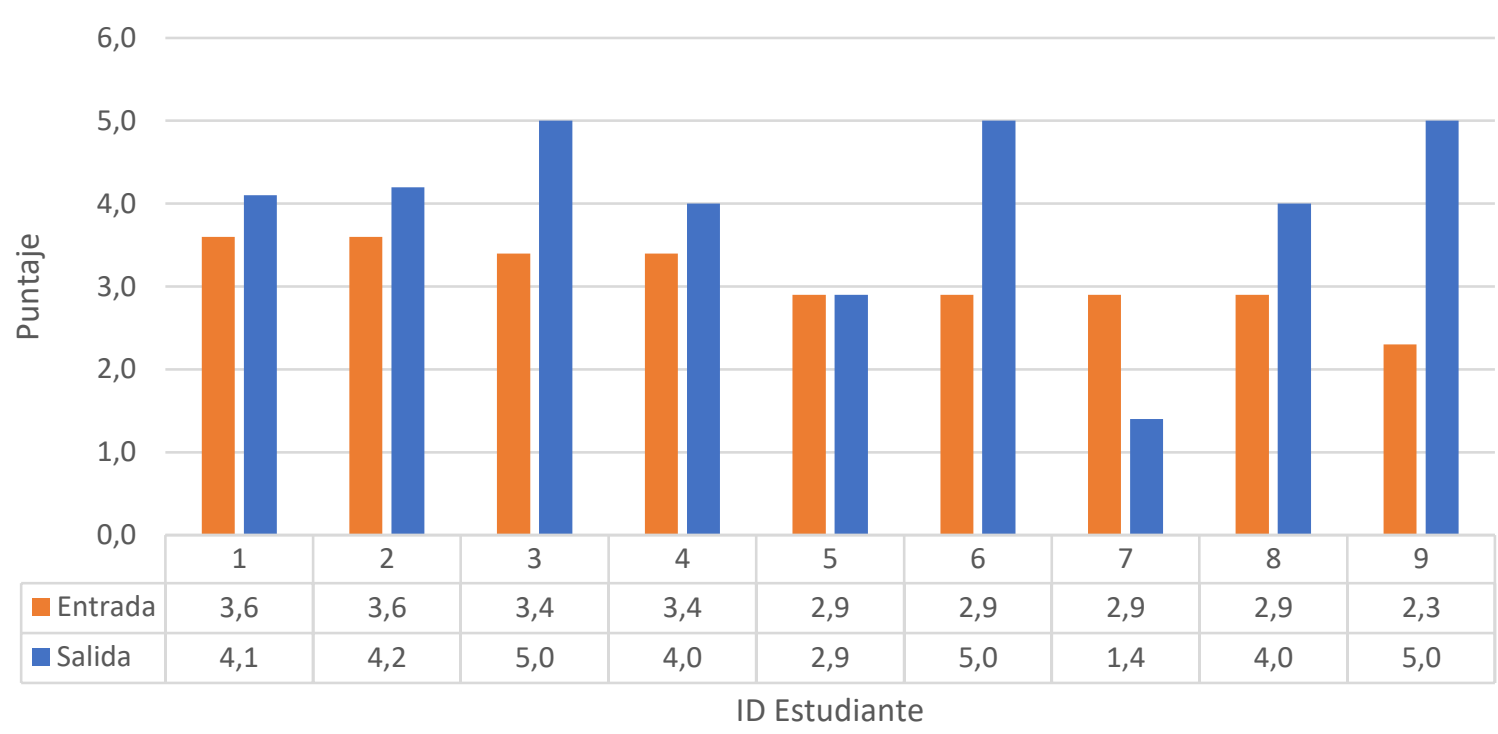

Nota. Progreso significativo del aprendizaje, elaboración propia (2021).

Por otra parte, de las entrevistas previstas, solo se aplicaron 5 al iniciar la intervención y 5 al finalizar la misma, debido a dificultades de conexión y disponibilidad de los estudiantes que decidieron desarrollar su proyecto de aula en torno a la respiración celular. Los estudiantes expresaron haber mejorado sus habilidades y su disposición hacia las herramientas y tecnologías que encontraron a su disposición para mejorar su proceso de aprendizaje. De igual manera, se pudo evidenciar mayor uso y diversidad de páginas web, así como, de otro tipo de recursos de la web 2.0 al momento de elaborar entregas y compromisos para la universidad.
Por otra parte, la mayoría de los estudiantes consideran haber construido una red de aprendizaje durante la intervención conectivista y son conscientes de la importancia de ésta y de la necesidad de ampliarla, mantenerla y navegarla.

Finalmente, es importante resaltar que los estudiantes confeccionaron un artículo de revisión en torno a la respiración celular y su relación con ciertos procesos fisiológicos humanos, puntualmente, sobre el efecto Warburg y su relación con las células cancerígenas, la fatiga muscular en 
deportistas y los efectos del material particulado presente en el aire. Adicional a este producto, los estudiantes construyeron un Blog (ver Figura 3) como herramienta para consolidar y divulgar la información revisada sobre la respiración y además como medio para interactuar con personas interesadas en la respiración celular a nivel mundial. El enlace y algunas piezas visuales fueron compartidas a través de las redes sociales de los estudiantes para alcanzar mayor impacto y visibilidad.

\title{
Figura 3
}

Blog Efecto Warburg: Respiración Celular y Metabolismo en Células Cancerígenas

\section{FATIGA MUSCULAR}

\begin{abstract}
Se manifiesta por descenso de la capacidad de generar potencia y se da en contracciones máximas o submáximas. Definida "una incapacidad para mantener los requerimientos o la fuerza generada"
\end{abstract}

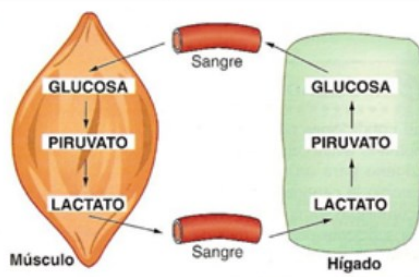

PRINCIPALES CAUSAS DE LA FATIGA MUSCULAR:

\section{- Las alteraciones del $\mathrm{pH}$, de la temperatura y del flujo sanguíneo, la acumulación de productos del metabolismo celular. -La pérdida de la homeóstasis del ion $\mathrm{Ca} 2+$. -La lesión muscular y el stress oxidativo.}
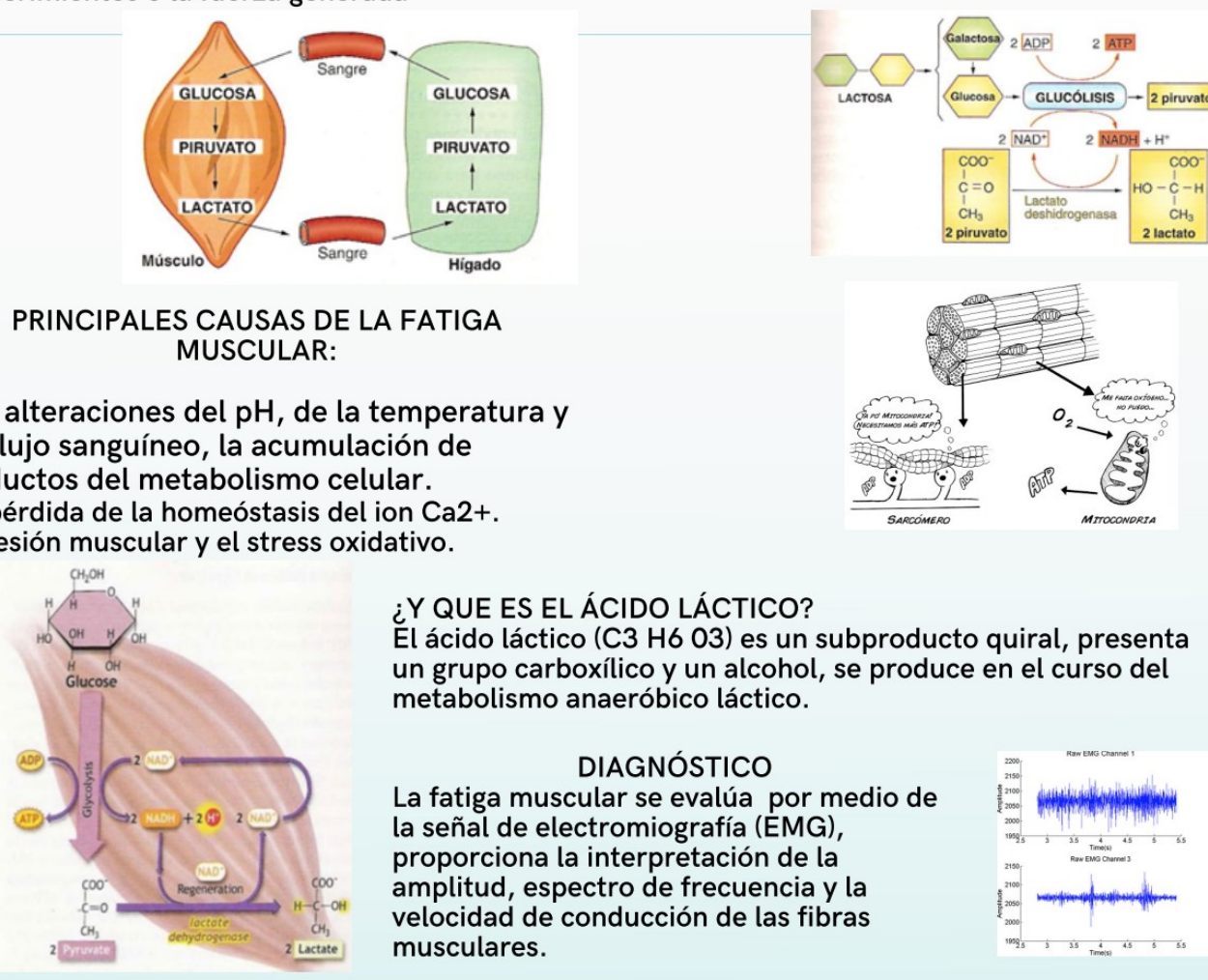

Nota. Actividad realizada con recursos 2.0, elaborado por los estudiantes (2021).

\section{Conclusiones}

Las herramientas tecnológicas bien aplicadas en el proceso de aprendizaje de asignaturas como bioquímica o biología molecular, pueden captar la atención del estudiante, motivarlo y permitirle aprender, como, además, retener en mayor medida la información. La construcción de redes de aprendizaje desarrolla habilidades y competencias en los estudiantes que las implementan como parte de su proceso de aprendizaje, abren su visión académica y personal. Como plantea Bartolomé (2011), aprender es el proceso por el cual el sujeto enriquece su red y el flujo de conocimiento a través de ella.

El conectivismo facilitó y motivo a los estudiantes a explorar las herramientas que tiene a su disposición y desconoce, los invito a construir su propia red de aprendizaje y a gestionar su proceso formativo. Los aportes del conectivismo a asignaturas complejas como bioquímica y biología molecular son claros, pues "el aprendizaje es un proceso de 
crecimiento y desarrollo, no solo de adquisición y creación" (Vadillo-Bueno, 2018). Sin embargo, se deben implementar otras estrategias enmarcadas en el conectivismo para conocer sus alcances $\mathrm{y}$ potenciales aportes.

De acuerdo con Bernal (2019), diseñar propuestas pedagógicas conscientes y coherentes con las características innovadores de la educación como el conectivismo, son necesarias en el contexto de la formación universitaria, pues se debe desarrollar en los estudiantes competencias acordes con la sociedad del siglo XXI.

Es importante continuar estudiando los potenciales aportes del conectivismo al proceso de aprendizaje de otras asignaturas y contenidos propios de las áreas de la salud, que dada su complejidad y vertiginoso avance, requieren una transformación. Las redes sociales, su fácil acceso y ubicuidad, pueden considerarse como herramientas útiles para la construcción de la red de aprendizaje de los estudiantes, pues facilita la conexión entre divulgadores científicos, creadores de contenido, expertos y aprendices de diversas áreas del conocimiento.

El conectivismo es una alternativa que merece ser explorada en las instituciones de educación superior, sin confrontar la educación formal y sin alterar la fundamentación metodológica y las restricciones que se imponen a los docentes en las aulas (Altamirano, 2010).

\section{Referencias}

Altamirano-Carmona, E.; Becerra-Correa, N. \& NavaCasarrubias, A. (2010). Hacia una Educación Conectivista. Revista Alternativa. 22 http://www.revistaalternativa.org

Bartolomé, A. (2011). Conectivismo. Aprender en red y en La red. A. Bartolomé, Teconologias na educação: Uma abordagem crítica para uma atualização prática.

Bernal, E. (2019). El conectivismo y su aplicación a través de herramientas Web 2.0: configuración de una red de aprendizaje para la producción de artículos científicos.

http://repository.udistrital.edu.co/bitstream/11349/1 4888/1/BernalGarzonEileen2019.pdf

Campbell, P.N. (1975) Teaching Biochemistry to Medical Students. Biochemical Education Vol 3 (1)
Downes, S. (2005). An Introduction to Connective Knowledge. bin/page.cgi?post=33034

Downes, S. (2006). Connectivism and Connective Knowledge Essays on meaning and learning networks. (National Research Council Canada, Ed.).

Downes, S. (2010). New technology supporting informal learning. Journal of Emerging Technologies in Web Intelligence, 2(1), 27-33.

Helle, L., Tynjälä, P. \& Olkinuora, E. (2006) Project-Based Learning in Post-Secondary Education - Theory, Practice and Rubber Sling Shots. High Educ 51, 287-314. https://doi.org/10.1007/s10734004-6386-5

Hernández-Sampieri, R.; Fernández-Collado \& C; BaptistaLucio, P. (2006). Metodología de la Investigación. Cuarta Edición. McGraw-Hill Interamericana.

Koppenol, W. H., Bounds, P. L., \& Dang, C. V. (2011). Otto warburg's contributions to current concepts of cancer metabolism. Nature Reviews Cancer, 11(5), 325-337. doi:10.1038/nrc3038

Lucumí-Moreno, A. (2015). Retos de la enseñanza de Biología Molecular y Bioquímica en las Carreras del Área de la Salud. Boletín Virtual Vol 4 (9). ISNN: 2266-1536.

Muñoz-Cano, J. M \& Maldonado-Salazar, T. N. J. (2011). Aprendizaje en medicina con base en proyectos. Una estrategia de metacognición. Perspectivas Docentes 45. Acotaciones.

Rodrigo-Mendizábal, I.F, López-Jimenez, D.F. \& ArribasUrrutia, A. (2020). Youth internet consumption in Ecuador: indicators of the national digital generation. Int. J. Web Based Communities. Vol. 16 (3). 296-320. http://dspace.uhemisferios.edu.ec:8080/xmlui/handl e/123456789/1189

Saldaña-Acosta, J. (2021). Desarrollo de Actitudes de Colaboración, Cooperación e Innovadoras en el Aula Basado en Proyectos. Revista TecnológicaEducativa Docentes 2.0, 11(1), 130-139. https://doi.org/10.37843/rted.v11i1.201

Siemens, G. (2004). Connectivism: A Learning Theory for the Digital Age. http://www.elearnspace.org/Articles/connectivism.ht $\mathrm{m}$

Siemens, G. (2005). Connectivism: A learning theory for the digital age. International Journal of Instructional Technology and Distance Learning, 2(1), 3-10.

Siemens, G., Leal, D. (2007). Conectivismo: Una teoría de aprendizaje para la era digital. En D. Siemens, G., Leal, Conectados en el ciberespacio.

Strauss, A. \& Corbin, J. (2002). Bases de la Investigación Cualitativa. Técnicas y Procedimientos para Desarrollar la Teoría Fundamentada. Primera 
Edición en español. Editorial Universidad de Antioquia.

Valerio-Ureña, G., \& Valenzuela-González, J.R. (2011). Contactos de redes sociales en línea como repositorios de información. RUSC. Universities and Knowledge Society Journal, 8(1),128-14. https://www.redalyc.org/articulo.oa?id=7801712600 6

Vadillo-Bueno, G. (2018). Stephen Downes y el conectivismo. Revista Mexicana De Bachillerato A Distancia, 10(19), 3 doi:http://dx.doi.org/10.22201/cuaed.20074751e.201 8.19.64909

Wood, E (2001). Biochemistry and molecular biology teaching over the past 50 years. Nat Rev Mol Cell Biol 2, 217-221. doi:10.1038/35056600 